\title{
Heating by absorption in the focus of an objective lens
}

\author{
Andreas Schönle and Stefan W. Hell \\ High Resolution Optical Microscopy Group, Max-Planck-Institute for Biophysical Chemistry, D-37070 Göttingen, Germany
}

Received December 8, 1997

\begin{abstract}
We derive an integral solution for the local heating of a linearly absorbing, uniform medium exposed to strongly focused light. Numerical results for local heating under typical multiphoton microscopy and optical trapping conditions are presented for various aperture angles. In contrast with common Gaussian beam approximations, our model employs the focal-intensity distribution as described by the point spread function of the lens. In this way, the model also accounts for axial heat transportation, which results in a lower prediction for the temperature increase. For an aperture of 1.2 (water immersion), irradiation with $100 \mathrm{~mW}$ of $850-\mathrm{nm}$ light for $1 \mathrm{~s}$ increases the local temperature of water by $0.2 \mathrm{~K}$. Heating of water by linear absorption can be ruled out as a limiting factor in standard multiphoton-excitation microscopy. (C) 1998 Optical Society of America
\end{abstract}

OCIS codes: $\quad 180.0180,300.1030,020.4180$

Focal heating caused by linear absorption in the near infrared is receiving considerable attention as a limiting factor in the rapidly evolving techniques of optical trapping ${ }^{1}$ and multiphoton-excitation microscopy. ${ }^{2}$ Viable biological specimens consist mainly of water, which because it features nonnegligible absorption in the 700-1100-nm wavelength range ${ }^{1}$ is expected to be heated in the sample. Two-photon microscopy is therefore usually performed with subpicosecond lasers, which provide high peak power at moderate average power, ${ }^{2} P_{\text {ave }}$. The signal of a two-photon-excitation fluorescence microscope varies with $P^{2}$ ave $/(\tau f)$, where $\tau$ represents the pulse width and $f$ the repetition rate. ${ }^{3}$ For high numerical apertures (NA's) of 1.0-1.2 (for water), $\tau=200 \mathrm{fs}$, and $f=80 \mathrm{MHz}$, a rather modest $P_{\text {ave }} \approx 3-15 \mathrm{~mW}$ is used. Pulse compression often is employed to reduce further the average power used.

Useful estimates of focal heating have been published, ${ }^{2,4}$ al beit for simplified conditions. Experimental results ${ }^{5}$ obtained for optical tweezer conditions showed sample heating of slightly less than $3 \mathrm{~K}$ at $\mathrm{NA}=1.3$ (oil) and $P_{\mathrm{ave}}=170 \mathrm{~mW}$. Denk et al. ${ }^{2}$ estimated the temperature increase in femtosecond two-photon-excitation microscopy and predicted a temperature increase of water in the range of as much as a few degrees Kelvin for $P_{\text {ave }}=50 \mathrm{~mW}$. This model implied an axially invariant Gaussian focus that, besides not representing high-NA conditions, cannot account for axial heat transportation. In essence, heating through linear absorption (of water) remains an issue in multiphoton-excitation microscopy. In this Letter we present a method based on the three-dimensional intensity point spread function (PSF) of the lens that allows for precise calculation of the focal temperature increase.

Let us assume an infinite sample with uniform extinction coefficient $\gamma$, volume heat capacity $c_{\nu}$, heat conductivity $\Lambda$, and refractive index $n$. The temporal evolution of the temperature $T$ is then given by the differential equation

$$
\left(\partial_{t}-C \nabla^{2}\right) T(\mathbf{r}, t)=f(\mathbf{r}, t),
$$

where $C=\Lambda / c_{\nu}, f=p / c_{\nu}$, and $p$ denotes the absorbed power per unit volume. Let $r$ and $z$ be cylindrical coordinates originating at the focal point and $\nu=$ $k \times \mathrm{NA} \times \mathrm{r}$ and $u=k \times a \times \mathrm{NA} \times z$ the corresponding optical coordinates. Here the NA is $n \sin (\alpha), n$ is the refractive index of the medium, $\alpha$ is the semi-aperture angle, $k=2 \pi / \lambda$, and $\lambda$ is the vacuum wavelength. For convenience we define $a=4 \sin ^{2}(\alpha / 2) / \sin (\alpha)$. We describe the focal-light distribution with the dimensionless intensity PSF $h(u, \nu)$ and introduce a new time scale, $\beta=t / \beta_{0}$, with $\beta_{0}=\left[k^{2}(\mathrm{NA})^{2} C\right]^{-1}$. With $h(u, \nu)$ normalized to unity, the integral over a plane of constant $u$ is well approximated by $4 \pi$. We then rewrite Eq. (1) as follows:

$$
\left(\partial_{\beta}-a^{2} \partial_{u}^{2}-\frac{1}{\nu} \partial_{\nu} \nu \partial_{\nu}\right) \vartheta=h
$$

where $T=\xi \vartheta=\gamma P / 4 \pi \Lambda \vartheta$. An integral solution can readily be derived from $\mathrm{Eq}$. (2), but a numerical analysis involving long irradiation times becomes inaccurate because of the oscillating nature of the PSF and the finite integration range. An upper-bound approximation can be given with an axially invariant Gaussian beam, $h(\nu)=\exp \left(-\nu^{2} / 4\right)$, and, neglecting the axial heat conduction, we find that $\vartheta^{\prime}(\beta)=\ln (1+\beta)$.

To derive the exact solution, we Fourier transform Eq. (2) in the space coordinates:

$$
\left(\partial_{\beta}+l^{2}+a^{2} s^{2}\right) \hat{\vartheta}(l, s)=c(l, s) .
$$

Here $c(l, s)=\int \mathrm{d} v v \int \mathrm{d} u \exp (i u s) J_{0}(l v) h(v, u)$ is the optical transfer function for which a closed-form expression is found that involves one incomplete elliptical integral of the second kind. ${ }^{6} c(l, s)$ has a first-order singularity in $l$ at the origin and is zero for $l>2$ or $s>0.5$. Note that $l$ and $s$ are different in Ref. 6. Temporal dependence of the irradiation can be accounted for by a $\beta$-dependent complex factor on 
the right-hand side of Eq. (3). For a sudden onset of radiation at $\beta=0$ the solution is given by

$$
\begin{aligned}
\hat{\vartheta}(l, s, \beta)= & c(l, s) \int_{0}^{\beta} \mathrm{d} \beta^{\prime} \exp \left[\left(-l^{2}-a^{2} s^{2}\right)\left(\beta-\beta^{\prime}\right)\right], \\
= & c(l, s)\left(l^{2}+a^{2} s^{2}\right)^{-1} \\
& \times\left\{1-\exp \left[\left(-l^{2}-a^{2} s^{2}\right) \beta\right]\right\}
\end{aligned}
$$

We readily find $\vartheta$ by calculating the Fourier backtransform of $\hat{\vartheta}$. The temperature is thus given by $1 / 2 \pi$ times the volume integral of $\hat{\vartheta}$, where the integrand turns nonsingular in the origin owing to the two-dimensional integral over $l$. Note that $\vartheta$ depends solely on the aperture angle.

Numerical evaluation was done with an algorithm that integrated $\hat{\vartheta}$ over the space of positive $l$ and $s$. The integration area was successively contracted around the origin. Figure 1 shows the temporal evolution of focal heating for a NA $=1.2$ water-immersion lens calculated with a relative accuracy of $0.4 \%$, along with the prediction made with the Gaussian beam approximation. It is found that although the logarithmic behavior is still valid the calculation for the Gaussian beam significantly overestimates the temperature increase.

This overestimation is expected to be greatest for high apertures, at which, owing to inherent strong axial focusing, axial heat dissipation is important. This expectation is confirmed in the calculations (Table 1 ), in which the temperature increase predicted by the Gaussian model is compared with that predicted by our model. Table 1 shows the focal-temperature increase in water exposed to constant 850-nm irradiation of $P_{\text {ave }}=100 \mathrm{~mW}$ of average power for $1 \mathrm{~s}$. The Gaussian approximation overestimates focal heating by 35$55 \%$ for very high NA's (1.2-1.3). In fact, the focal temperature increase is less than $0.25 \mathrm{~K}$ for all NA's considered.

It is also interesting to consider the limiting case in which the irradiation time approaches zero. In this case, heat dissipation becomes too slow to cool off the focal area. Heating then depends solely on $P_{\text {ave }}$; i.e., the Gaussian model becomes accurate. The temperature increase will be stronger for a higher NA and can be estimated with $\vartheta=\xi t / \beta_{0}$. In particular we can estimate transient heating by a single pulse of duration $\tau$, as encountered in multiphoton-excitation microscopy. For pulses in the femotosecond and picosecond ranges, the increase in temperature does not depend on $\tau$ and is given by $\Delta T=\xi /\left(\beta_{0} v\right)$. Assuming that $\Lambda=0.6 \mathrm{~W} \mathrm{~K}^{-1} \mathrm{~m}^{-1}$ and $\gamma=1.3 \mathrm{~m}^{-1}$, we obtain $\Delta T=0.0025 \mathrm{~K}$ for a Ti:sapphire system running at $850 \mathrm{~nm}$.

Table 2 shows the result of irradiation at NA $=1.2$ at different wavelengths for $1 \mathrm{~ms}, 1 \mu \mathrm{s}, 1 \mathrm{~s}$, and $10 \mathrm{~s}$, thus accounting for the spectral dependence of the extinction coefficient. Focal heating owing to linear absorption by water can be expected to remain less than $3 \mathrm{~K}$ for all wavelengths shown. An estimate for arbitrary irradiation times, wavelengths, or media can be derived with Tables 1 and 2 in conjunction with Fig. 1.

Our calculation implies a sample with infinite boundaries. The sample, however, is in contact with the coverslip and the microscope, which can act as a heat sink. By displaying a (slow) logarithmic increase, the calculations slightly overestimate the longterm heating. Even for constant irradiation the sample temperature is expected to approach a steadystate value. However, we do not expect this phenomenon to be significant in image recording because of the short times involved.

Transient heating by two-photon absorption is sometimes mentioned as a potential hazard. The squaredintensity PSF describing two-photon absorption can be well approximated by a double Gaussian, so a closedform solution is readily found. We note that, given

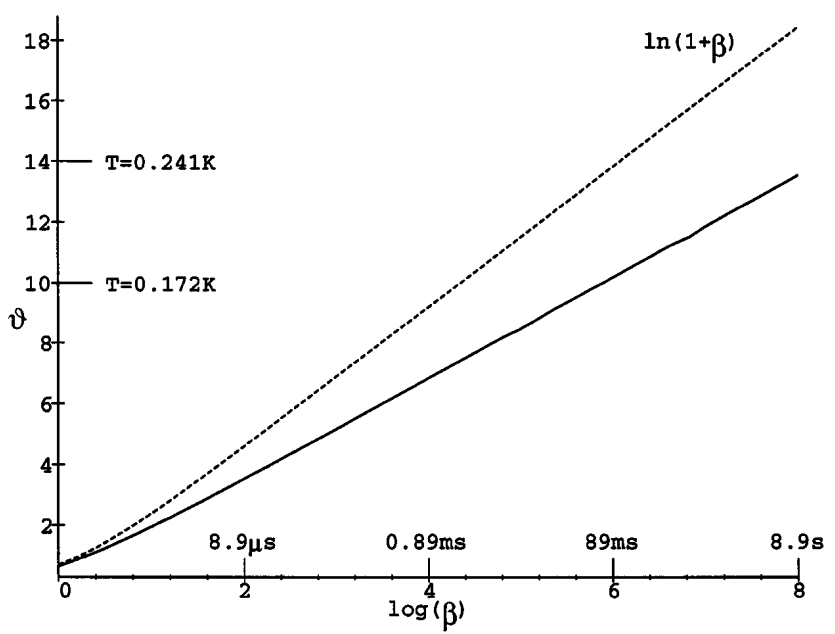

Fig. 1. Temperature increase of the focal point of a lens focusing light into a uniform medium, as predicted by the Gaussian beam approximation (dashed curve) and numerical calculation (solid curve). The curves can be applied to various media and wavelengths by use of appropriate scaling. The inserted time scale and temperature scale exemplify the case of a NA $=1.2$ water-immersion lens focusing $100 \mathrm{~mW}$ of $850-\mathrm{nm}$ light into water.

Table 1. Temperature Increase $T$ and Logarithmic Approximation $T^{*}$ for Different NA's after Irradiation of Water with $100 \mathrm{~mW}$ at $850 \mathrm{~nm}$ for $1 \mathrm{~s}$

\begin{tabular}{ccccc}
\hline NA & $\beta_{0}(\mathrm{~ns})$ & $T(K)$ & $T^{*} K$ & $T / T^{*}-1(\%)$ \\
\hline 0.4 & 800 & 0.231 & 0.242 & 4.7 \\
0.8 & 200 & 0.236 & 0.265 & 12.3 \\
1.0 & 128 & 0.227 & 0.273 & 20.3 \\
1.2 & 89 & 0.206 & 0.279 & 35.4 \\
1.3 & 76 & 0.183 & 0.282 & 54.1 \\
\hline
\end{tabular}

Table 2. Temperature Increase for Different Wavelengths at $\mathrm{NA}=1.2$ after Irradiation of Water with $100 \mathrm{~mW}$ for Different Durations

\begin{tabular}{rcccccc}
\hline & & & \multicolumn{4}{c}{$T(\mathrm{~K})$} \\
\cline { 4 - 7 }$\lambda(\mathrm{nm})$ & $\xi(\mathrm{K})$ & $\beta_{0}(\mathrm{~ns})$ & $10 \mathrm{~s}$ & $1 \mathrm{~s}$ & $1 \mathrm{~ms}$ & $1 \mu \mathrm{s}$ \\
\hline 650 & 0.013 & 52 & 0.18 & 0.16 & 0.10 & 0.02 \\
750 & 0.016 & 69 & 0.22 & 0.19 & 0.11 & 0.04 \\
850 & 0.017 & 89 & 0.23 & 0.21 & 0.12 & 0.04 \\
950 & 0.172 & 111 & 2.32 & 2.03 & 1.16 & 0.33 \\
1050 & 0.146 & 135 & 1.94 & 1.69 & 0.97 & 0.26 \\
\hline
\end{tabular}


a micromolar absorber concentration and a $20 \%$ excitation rate of molecules per pulse, we calculate an upper bound for the temperature increase in the submillikelvin range if all absorbed power is transformed into heat.

Liu et al. ${ }^{5}$ measured a temperature increase of $<3 \mathrm{~K}$ after 8-s irradiation of an aqueous sample by $P_{\text {ave }}=$ $170 \mathrm{~mW}$ of 1064-nm laser light focused with a NA $=1.3$ oil-immersion lens. The aperture angle is comparable with that of a NA $=1.14$ water-immersion lens, for which we calculate a temperature increase of $3.4 \mathrm{~K}$, in close agreement with the experimental result. The residual difference can be explained by the large refractive-index mismatch between the oil-immersion system and the water sample. ${ }^{7}$

Importantly, our results imply that, for a large power, $P_{\text {ave }} \approx 100 \mathrm{~mW}$, and $\lambda<1070 \mathrm{~nm}$, the water temperature in the focus will not increase more than $3 \mathrm{~K}$. Hence, for most multiphoton microscopy applications, heating through linear absorption will not play a destructive role, except in (probably rare) cases in which other strongly absorbing molecules are present in high concentrations. This conclusion holds for pulsed and cw lasers. We believe that our findings will encourage the development of two-photon microscopy with longer pulses and cw beams. For a higher average power, optical trapping in the sample cannot be ignored. Our results, however, show that heating of water by linear absorption can be ruled out as a limiting phenomenon in standard multiphoton microscopy.

\section{References}

1. K. Svoboda and S. M. Block, Annu. Rev. Biophys. Biomol. Struct. 23, 247 (1994).

2. W. Denk, D. W. Piston, and W. W. Webb, in Two-Photon Molecular Excitation in Laser Scanning Microscopy, J . B. Pawley, ed. (Plenum, New York, 1995), p. 445- 458.

3. P. E. Hänninen, E. Soini, and S. W. Hell, J. Microsc. 176, 222 (1994).

4. A. Sennaroglu, J. Opt. Soc. Am. B 14, 356 (1997).

5. Y. Liu, D. K. Cheng, G. J. Sonek, M. W. Berns, C. F. Chapman, and B. J. Tromberg, Biophys. J. 68, 2137 (1995).

6. M. Gu, Principles of Three-Dimensional Imaging in Confocal Microscopes (World Scientific, Singapore, 1996), p. 277.

7. S. W. Hell, G. Reiner, C. Cremer, and E. H. K. Stelzer, J. Microsc. 169, 391 (1993). 